Proceedings of the Edinburgh Mathematical Society (2004) 47, 375-395 (C)

DOI:10.1017/S0013091503000658 Printed in the United Kingdom

\title{
ASYMPTOTIC BEHAVIOUR AND BLOW-UP FOR A NONLINEAR DIFFUSION PROBLEM WITH A NON-LOCAL SOURCE TERM
}

\author{
N. I. KAVALLARIS \\ Department of Mathematics, Faculty of Applied Sciences, \\ National Technical University of Athens, Zografu Campus, \\ 15780 Athens, Greece (nkaval@math.ntua.gr)
}

(Received 21 July 2003)

Abstract In this work, the behaviour of solutions for the Dirichlet problem of the non-local equation

$$
u_{t}=\Delta(\kappa(u))+\frac{\lambda f(u)}{\left(\int_{\Omega} f(u) \mathrm{d} x\right)^{p}}, \quad \Omega \subset \mathbb{R}^{N}, \quad N=1,2,
$$

is studied, mainly for the case where $f(s)=\mathrm{e}^{\kappa(s)}$. More precisely, the interplay of exponent $p$ of the non-local term and spatial dimension $N$ is investigated with regard to the existence and non-existence of solutions of the associated steady-state problem as well as the global existence and finite-time blow-up of the time-dependent solutions $u(x, t)$. The asymptotic stability of the steady-state solutions is also studied.

Keywords: non-local parabolic problems; asymptotic behaviour; blow-up

2000 Mathematics subject classification: Primary 35K60

Secondary 35B40

\section{Introduction}

In this paper, existence and non-existence results are obtained for solutions of initialboundary-value problems to the non-local parabolic equation

$$
u_{t}-\Delta(\kappa(u))=\frac{\lambda f(u)}{\left(\int_{\Omega} f(u) \mathrm{d} x\right)^{p}}, \quad x \in \Omega, \quad t>0 .
$$

Here $f(u)$ is a Lipschitz continuous, positive, increasing function and $\kappa(u)$ is imposed to be a positive function with $\kappa \in C^{2}\left(\mathbb{R}_{+}\right) . \Omega$ is a smooth bounded subset of $\mathbb{R}^{N}$ and $\lambda, p$ are positive parameters. Also, for simplicity, it is assumed that $u(x, 0)=\psi(x)$ is continuous with $\psi(x)=0, x \in \partial \Omega$ and $\psi(x) \geqslant 0, x \in \Omega$ (the last may be supposed, without loss of generality, as a consequence of the fact that for any $\psi(x)$ the solution $u(x, t)$ becomes non-negative through $\Omega$ at some time $t)$.

Our original motivation for studying such problems comes from the plasma ohmic heating process. The plasma is an electrical conductor and so it could be heated by passing 
a current through it. This is called ohmic heating and it is the same kind of heating that occurs in thermistors. One naturally encounters, in one dimension, an equation of the form

$$
u_{t}=\left(u^{4}\right)_{x x}+\frac{\lambda g(u)}{\left(\int_{-1}^{1} g(u) \mathrm{d} x\right)^{2}}, \quad-1<x<1, \quad t>0,
$$

where $u=u(x, t)$ stands for the dimensionless temperature of the plasma, while $g(u)$ represents the temperature-dependent electrical resistance of plasma (see $[\mathbf{1 3}, \mathbf{1 4}])$. The nonlinear diffusion term $\left(u^{4}\right)_{x x}$ comes from assuming the Stefan-Boltzmann law for emission of thermal radiation. However, it is possible that some more complicated expression, depending on the nature of heat transport, could replace $\left(u^{4}\right)_{x x}$. Moreover, the non-local term $\lambda g(u) /\left(\int_{-1}^{1} g(u) \mathrm{d} x\right)^{2}$ arises due to the ohmic heating nature of the process. For more details concerning the derivation of the mathematical model (1.2), see [13] and the references cited therein.

The key for the study of the asymptotic behaviour of the solutions to Equation (1.1) is the study of the associated steady-state equation

$$
-\Delta(\kappa(w))=\frac{\lambda f(w)}{\left(\int_{\Omega} f(w) \mathrm{d} x\right)^{p}}, \quad x \in \Omega
$$

Our main purpose, in this work, is to show the interplay of $p$, the exponent of the non-local term, the non-local parameter $\lambda$ and the spatial dimension $N$, with regard to the existence or non-existence of solutions to Equation (1.3) and the global existence or blow-up of solutions to Equation (1.1). The study of the asymptotic behaviour of solutions to Equation (1.1), when $f$ is a decreasing function, can be undertaken, since a maximum principle holds, by using comparison techniques $[\mathbf{1 3}, \mathbf{1 4}]$. However, when $f$ is an increasing function, comparison techniques fail, because there is not a valid maximum principle for Equation (1.1). In this case, the asymptotic behaviour can be studied either with a dynamical-systems approach [4] or probably using eigenfunctions expansions [9].

We will place emphasis on cases where the spatial dimension $N$ is 1 or 2 , the nonlinearity $f(u)=\mathrm{e}^{\kappa(u)}$ and the boundary conditions are of Dirichlet type. In these cases, we study stability of solutions of Equation (1.3) by constructing a Lyapunov functional, which also helps us to study the global existence and blow-up of solutions to the timedependent problem.

Following a method due to Fila $[\mathbf{6}, \mathbf{7}]$ and some ideas of Lieberman $[\mathbf{1 2}]$, we extend some of the results of $[\mathbf{1 3}, \mathbf{1 4}]$, where $f(u)$ is now an increasing function and $\left(u^{4}\right)_{x x}$ is replaced by the more complicated nonlinear diffusion term $(\kappa(u))_{x x}$. Simultaneously, these results are extended to the case of spatial dimension $N=2$.

The paper is arranged as follows. In $\S 2$ we establish the local existence and uniqueness of solutions to the initial-boundary-value problem of Equation (1.1). In $\S 3$ we obtain existence-non-existence results of the steady-state problem. The questions of global existence and finite-time blow-up for solutions to initial-boundary-value problems for Equation (1.1) are addressed in $\S \S 4$ and 5 , respectively. 


\section{Existence and uniqueness}

In order to guarantee the existence of a solution for the problem

$$
\begin{gathered}
u_{t}=\Delta(\kappa(u))+\frac{\lambda f(u)}{\left(\int_{\Omega} f(u) \mathrm{d} x\right)^{p}}, \quad x \in \Omega, \quad t>0, \\
B(u(x, t))=0, \quad x \in \partial \Omega, \quad t>0, \\
u(x, 0)=\psi(x), \quad x \in \Omega,
\end{gathered}
$$

where $B$ is a boundary operator, we impose that $\kappa(s), \kappa^{\prime}(s)$ and $\kappa^{\prime \prime}(s)$ satisfy precisely one of the following conditions (see [11, Chapter V.1]):

$$
\kappa \in C^{2}\left(\mathbb{R}_{+}\right), \quad \kappa(s)>0, \quad \kappa^{\prime}(s)>0, \quad \kappa^{\prime \prime}(s)>0, \quad s \geqslant 0
$$

or

$$
\begin{aligned}
\kappa \in C^{2}\left(\mathbb{R}_{+}\right), \quad \kappa(s)>0, & \kappa^{\prime}(s)>0, \quad \kappa^{\prime \prime}(s)>0, \quad s>0 \\
& \text { and } \quad \kappa(0)=\kappa^{\prime}(0)=\kappa^{\prime \prime}(0)=0 .
\end{aligned}
$$

In contrast to the decreasing case, when $f(s)$ is an increasing function, the existence of an upper and a lower solution in the classical sense does not guarantee the existence of a solution of problem (2.1), which lies between them. In order to overcome this difficulty we introduce the concept of lower-upper solution pairs.

Definition 2.1. A pair of functions $(z, v)$ with $z, v \in C^{2,1}\left(\Omega_{T} ; \mathbb{R}\right) \cap C\left(\bar{\Omega}_{T} ; \mathbb{R}\right)\left(\Omega_{T}=\right.$ $\Omega \times(0, T))$ is called a lower-upper solution pair for problem $(2.1)$ if $z(x, t) \leqslant v(x, t)$ for $(x, t) \in \bar{\Omega}_{T}$ and

$$
\begin{gathered}
z_{t} \leqslant \Delta(\kappa(z))+\frac{\lambda f(z)}{\left(\int_{\Omega} f(v) \mathrm{d} x\right)^{p}}, \quad v_{t} \geqslant \Delta(\kappa(v))+\frac{\lambda f(v)}{\left(\int_{\Omega} f(z) \mathrm{d} x\right)^{p}}, \quad x \in \Omega, \quad 0<t<T, \\
B(z) \leqslant 0 \leqslant B(v), \quad x \in \partial \Omega, \quad 0<t<T, \\
z(x, 0) \leqslant \psi(x) \leqslant v(x, 0), \quad x \in \Omega .
\end{gathered}
$$

If all the above inequalities are strict, then $(z, v)$ is a strict lower-upper solution pair $($ see $[3,5])$.

Now let $(z, v)$ be a lower-upper solution pair and an iterative scheme starting with $\bar{u}_{0}=v, \underline{u}_{0}=z$ and proceeding according to

$$
\begin{gathered}
\underline{u}_{n t}=\Delta\left(\kappa\left(\underline{u}_{n}\right)\right)+\frac{\lambda f\left(\underline{u}_{n-1}\right)}{\left(\int_{\Omega} f\left(\bar{u}_{n-1}\right) \mathrm{d} x\right)^{p}}, \quad x \in \Omega, \quad t>0, \\
\bar{u}_{n t}=\Delta\left(\kappa\left(\bar{u}_{n}\right)\right)+\frac{\lambda f\left(\bar{u}_{n-1}\right)}{\left(\int_{\Omega} f\left(\underline{u}_{n-1}\right) \mathrm{d} x\right)^{p}}, \quad x \in \Omega, \quad t>0, \\
B\left(\underline{u}_{n}\right)=B\left(\bar{u}_{n}\right)=0, \\
\underline{u}_{n}(x, 0)=\bar{u}_{n}(x, 0)=\psi(x),
\end{gathered}
$$


for $n=1,2, \ldots$ The previous problems are local, hence using the maximum principle we see that $z \leqslant \underline{u}_{n-1} \leqslant \underline{u}_{n} \leqslant \bar{u}_{n-1} \leqslant \bar{u}_{n-1} \leqslant v$. So, we get two bounded monotonic sequences each of which converge to a solution of (2.1) as $n \rightarrow \infty$. Since $f$ is Lipschitz continuous we get that $u$ is unique and $z(x, t) \leqslant u(x, t) \leqslant v(x, t)$ for every $(x, t) \in \bar{\Omega}_{T_{\max }}$, where $T_{\max }$ is the maximal existence time for $u$. Thus we have proved the following existence theorem (see also $[\mathbf{3}, \mathbf{5}]$ ).

Proposition 2.2. Let $(z, v)$ be a lower-upper solution pair for (2.1). There then exists a unique solution $u$ to problem (2.1) such that

$$
z(x, t) \leqslant u(x, t) \leqslant v(x, t), \quad \text { for every }(x, t) \in \bar{\Omega}_{T_{\max }} .
$$

Remark 2.3. From the above analysis we get that the solution $u$ to $(2.1)$ continues to exist as long as it remains less than or equal to $b>\sup v(x, t)$. This argument implies that $u$ ceases to exist only by blow-up, i.e. if there exists a sequence $\left(x_{n}, t_{n}\right) \rightarrow\left(x^{*}, t^{*}\right)$ as $n \rightarrow \infty$ with $t^{*}<\infty$ such that $u\left(x_{n}, t_{n}\right) \rightarrow \infty$ as $n \rightarrow \infty$.

When $\kappa(s)$ satisfies condition (2.3) (e.g. $\kappa(s)=s^{m}, m>1$ ), then writing Equation $(2.1 a)$ in the form

$$
u_{t}=\kappa^{\prime}(u) \Delta u+\kappa^{\prime \prime}(u)|\nabla u|^{2}+\frac{\lambda f(u)}{\left(\int_{\Omega} f(u) \mathrm{d} x\right)^{p}},
$$

we obtain that this equation is (uniformly) parabolic for $u>0$, while it becomes degenerate when $u=0$. The degenerate problem does not admit a classical solution and so, in this case, the concept of the solution of (2.1) must be extended in some sense (see [6]). However, since $\psi(x)>0$, the maximum principle implies that $u(x, t)>0$ in $\bar{\Omega}_{T_{\max }}$. Under this hypothesis, problem (2.1) is non-degenerate and so always admits a classical solution, as was proved above.

\section{Steady-state problem}

The corresponding steady-state problem to (2.1) with Dirichlet boundary conditions is

$$
\begin{gathered}
\Delta(\kappa(w))+\frac{\lambda f(w)}{\left(\int_{\Omega} f(w) \mathrm{d} x\right)^{p}}=0, \quad x \in \Omega, \quad \Omega \subset \mathbb{R}^{N}, \\
w=0, \quad x \in \partial \Omega .
\end{gathered}
$$

Definition 3.1. While we will write (3.1) in the above form, $w \geqslant 0$ is called a solution to (3.1) exactly when $v=\kappa(w)$ is a classical solution $\left(v \in C^{2}(\Omega) \cap C^{1}(\bar{\Omega})\right)$ to the problem

$$
\begin{gathered}
\Delta v+\frac{\lambda g(v)}{\left(\int_{\Omega} g(v) \mathrm{d} x\right)^{p}}=0, \quad x \in \Omega, \\
v=\kappa(0), \quad x \in \partial \Omega,
\end{gathered}
$$

where $g(s)=f\left(\kappa^{-1}(s)\right)$. 
We start our study of the steady-state problem from the one-dimensional case.

Proposition 3.2. Let $N=1, \Omega=(-1,1)$ and $p=2$. Problem (3.1) that has a unique solution for every $\lambda>0$.

Proof. In this case, problem (3.1) takes the form

$$
\begin{gathered}
(\kappa(w))_{x x}+\frac{\lambda f(w)}{\left(\int_{-1}^{1} f(w) \mathrm{d} x\right)^{2}}=0, \quad-1<x<1, \\
w(-1)=w(1)=0
\end{gathered}
$$

and $v=\kappa(w)$ satisfies the problem

$$
\begin{gathered}
v^{\prime \prime}+\mu g(v)=0, \quad-1<x<1, \\
v(-1)=v(1)=\kappa(0)>0,
\end{gathered}
$$

with $\mu=\lambda /\left(\int_{-1}^{1} g(v) \mathrm{d} x\right)^{2}$. Integrating Equation $(3.4 a)$ over $(-1,1)$ and using the fact that $v$ is an even function, which comes from

$$
\int_{\kappa(0)}^{v(x)} \frac{\mathrm{d} \sigma}{\sqrt{G(M)-G(\sigma)}}=\int_{\kappa(0)}^{v(-x)} \frac{\mathrm{d} \sigma}{\sqrt{G(M)-G(\sigma)}}, \quad G(M)=\int_{\kappa(0)}^{M} g(s) \mathrm{d} s,
$$

we get

$$
\left(v^{\prime}(1)\right)^{2}=\frac{1}{4} \mu^{2}\left(\int_{-1}^{1} g(v) \mathrm{d} x\right)^{2} .
$$

Also, multiplying $(3.4 a)$ by $v^{\prime}$ and integrating over $(-1,1)$ we obtain

$$
\left(v^{\prime}(1)\right)^{2}=2 \mu \int_{\kappa(0)}^{M} g(s) \mathrm{d} s
$$

where $M=v(0)=\max _{[-1,1]} v(x)$. Now, combining (3.5) and (3.6) we take $\lambda(M) \equiv$ $\lambda=8 \int_{\kappa(0)}^{M} g(s) \mathrm{d} s$, which implies that $\lambda^{\prime}(M)=8 g(M)>0$ and hence $\lambda(M) \rightarrow \infty$ as $M \rightarrow \infty\left(g^{\prime}(s)>0, s>0\right.$, since $f(s)$ is increasing and either (2.2) or (2.3) hold, thus $\left.\int_{\kappa(0)}^{\infty} g(s) \mathrm{d} s=\infty\right)$; this completes the proof.

Below we give some results for the case where $f(s)=\mathrm{e}^{\kappa(s)}$.

Proposition 3.3. Let $N=1, f(s)=\mathrm{e}^{\kappa(s)}\left(g(s)=\mathrm{e}^{s}\right), \kappa(0)=0$ and $\Omega=(-1,1)$.

(a) If $p \geqslant 1$, then problem (3.3) has a unique solution for every $\lambda>0$.

(b) If $0<p<1$, then there exists $\lambda^{*}>0$ such that problem (3.3) has

(i) at least two solutions for $\lambda<\lambda^{*}$,

(ii) a unique solution for $\lambda=\lambda^{*}$, and

(iii) no solution for $\lambda>\lambda^{*}$. 
Proposition 3.4. Let $N=2, f(s)=\mathrm{e}^{\kappa(s)}\left(g(s)=\mathrm{e}^{s}\right), \kappa(0)=0$ and $\Omega=B(0,1)=$ $\left\{x \in \mathbb{R}^{2}:|x| \leqslant 1\right\}$.

(a) If $p>1$, then problem (3.1) has a unique solution for every $\lambda>0$.

(b) If $p=1$, then problem (3.1) has a unique solution for $0<\lambda<8 \pi$ and no solution for $\lambda \geqslant 8 \pi$.

(c) If $0<p<1$, then there exists $\lambda^{*}$ such that problem (3.1) has

(i) at least two solutions for $0<\lambda<\lambda^{*}$,

(ii) a unique solution for $\lambda=\lambda^{*}$, and

(iii) no solution for $\lambda>\lambda^{*}$.

The above propositions are proved in a similar way to Theorems 3.1 and 3.2 , respectively, in [4], also taking Definition 3.1 into account.

Also, using Pohozaev's identity, non-existence results can be extended to strictly starshaped domains. A domain $\Omega \subset \mathbb{R}^{N}, N \geqslant 2$, is strictly star-shaped (containing 0 ) if there exists $a>0$ such that

$$
x \cdot \eta \geqslant a \int_{\partial \Omega} \mathrm{d} s, \quad \text { for } \eta \text { unit outer normal. }
$$

It can be proved, provided $\int_{0}^{\infty} f(\sigma) \kappa^{\prime}(\sigma) \mathrm{d} \sigma<\infty$, that problem (3.1) has no solutions if $\lambda>2 N g^{p-2}(0)|\Omega|^{p-1} A(\partial \Omega) \int_{0}^{\infty} f(\sigma) \kappa^{\prime}(\sigma) \mathrm{d} \sigma / \alpha$ (see also [4]).

\section{Global existence and asymptotic stability}

We start this section with the one-dimensional case, giving a global existence result concerning the time-dependent problem for functions $f(s)$ that are bounded away from 0 .

Proposition 4.1. Let $f(s) \geqslant c>0$ and $p \geqslant 1$, then, for every positive bounded function $\psi(x)$ and $\lambda>0$, the problem

$$
\begin{gathered}
u_{t}=(\kappa(u))_{x x}+\frac{\lambda f(u)}{\left(\int_{\Omega} f(u) \mathrm{d} x\right)^{p}}, \quad x \in \Omega \subset \mathbb{R}, \quad t>0, \\
u(x, t)=0, \quad x \in \partial \Omega, \quad t>0, \\
u(x, 0)=\psi(x), \quad x \in \Omega,
\end{gathered}
$$

where $\Omega$ is a bounded subset of $\mathbb{R}$, has a (unique) uniformly bounded, classical solution in $Q_{\infty}=\bar{\Omega} \times[0, \infty)$.

Proof. Let

$$
g(x, t)=\frac{\lambda f(u)}{\left(\int_{\Omega} f(u) \mathrm{d} x\right)^{p}},
$$

then the following relation holds:

$$
\int_{\Omega} g(x, t) \mathrm{d} x=\frac{\lambda}{\left(\int_{\Omega} f(u) \mathrm{d} x\right)^{p-1}} \leqslant \frac{\lambda}{(c|\Omega|)^{p-1}}, \quad \text { for every } t>0,
$$

since $p \geqslant 1$. 
Using the maximum principle (see [2]) we take the estimate

$$
0 \leqslant u(x, t) \leqslant\|\psi\|_{\infty}+\Lambda \sup _{0<t<T}\|g(\cdot, t)\|_{L^{1}(\Omega)}, \quad \text { for every } t>0
$$

where $\Lambda$ is a constant depending only upon $|\Omega|$. Combining relations (4.2) and (4.3) we finally obtain the estimate

$$
0 \leqslant \sup _{0<t<T}\|u(\cdot, t)\|_{\infty} \leqslant\|\psi\|_{\infty}+\Lambda_{1}<\infty, \quad \text { for every } T>0
$$

with $\Lambda_{1}=2 \Lambda /(C|\Omega|)^{p-1}$. Due to the Nash-Moser Theorem and a Schauder-type estimate (see [11]), relation (4.4) leads to an estimate of the form

$$
\|u\|_{C^{2+\alpha, 1+\alpha / 2}\left(Q_{\infty}\right)} \leqslant \Lambda_{2}
$$

which establishes the existence of a classical global-in-time solution to problem (4.1).

Remark 4.2. In the case, where $f(s)$ is an increasing function, $f(s) \geqslant f(0)>0$ for $s \geqslant 0$, and so Proposition 4.1 is still valid.

Below we prove for the two-dimensional case $N=2$ a result analogous to Proposition 4.1, holding for problem (2.1) with Dirichlet boundary conditions, but only for $f(s)=\mathrm{e}^{\kappa(s)}$. This is because the proof is based on the existence of a Lyapunov functional for this problem and such a functional can be constructed only if $f(s)=\mathrm{e}^{\kappa(s)}$.

Indeed, for spatial dimensions $N=1,2$, problem (2.1) with Dirichlet boundary conditions generates a local semiflow in $L^{\infty}(\Omega) \cap H_{0}^{1}(\Omega)$ defined by

$$
S(t) \psi(\cdot)=u(\cdot, t ; \psi(x)), \quad \text { for } t>0 \text { and } \psi \in L^{\infty}(\Omega) \cap H_{0}^{1}(\Omega) .
$$

The (positive) orbit of the semiflow is $\gamma(\psi)=\{S(t) \psi: t \geqslant 0\}$ and its $\omega$-limit set is defined as

$$
\begin{array}{r}
\omega(\psi):=\left\{w \in L^{\infty}(\Omega): \text { there exists a sequence }\left(t_{n}\right)_{n \in \mathbb{N}}, t_{n} \rightarrow \infty\right. \text { such that } \\
\left.S\left(t_{n}\right) \psi \rightarrow w \text { in } C^{1}(\bar{\Omega}) \cap C^{2}(\Omega)\right\}
\end{array}
$$

in $C^{1}(\bar{\Omega}) \cap C^{2}(\Omega)$. For $p \neq 1$, this local semiflow has a Lyapunov functional given by

$$
J[v](t)=\frac{1}{2} \int_{\Omega}|\nabla \kappa(v)|^{2} \mathrm{~d} x+\frac{\lambda}{p-1}\left(\int_{\Omega} \mathrm{e}^{\kappa(v)} \mathrm{d} x\right)^{1-p}
$$

and the semiflow is gradient-like in the sense that for any $t \in\left[0, T_{\max }\right)$

$$
\int_{0}^{t} \int_{\Omega} \kappa^{\prime}(u) u_{t}^{2} \mathrm{~d} x \mathrm{~d} s+J[u](t)=J[\psi]
$$

Now we are ready to prove the following existence result. 
Proposition 4.3. Let $N=2$ and $p>1$. Then the problem

$$
\begin{gathered}
u_{t}=\Delta(\kappa(u))+\frac{\lambda \mathrm{e}^{\kappa(u)}}{\left(\int_{\Omega} \mathrm{e}^{\kappa(u)} \mathrm{d} x\right)^{p}}, \quad x \in \Omega, \quad t>0, \\
u(x, t)=0, \quad x \in \partial \Omega, \quad t>0 \\
u(x, 0)=\psi(x), \quad x \in \Omega
\end{gathered}
$$

where $\Omega$ is a smooth bounded subset of $\mathbb{R}^{2}$ and $\kappa(0)=0$ has a (unique) uniformly bounded solution in $Q_{\infty}=\bar{\Omega} \times[0, \infty)$, for every $\lambda>0$, provided that $\kappa(\psi) \in H_{0}^{1}(\Omega)$.

Proof. For the proof we proceed as in [4]. Let $u(x, t)$ be a classical solution to problem (4.6) in $\Omega \times[0, T)$. Then, due to (4.5), we have $J[u](t) \leqslant J[\psi]=E_{0}$, which for $p>1$ implies that $\int_{\Omega}|\nabla \kappa(u(x, t))|^{2} \mathrm{~d} x \leqslant 2 E_{0}$ for every $t>0$. From Gilbarg-Trudinger's inequality $[\mathbf{8}]$ we have

$$
\int_{\Omega} \exp \left[c_{1} \frac{|v|}{\|v\|}\right]^{2} \mathrm{~d} x \leqslant c_{2}|\Omega|, \quad \text { for every } v \in H_{0}^{1}(\Omega),
$$

where $c_{1}, c_{2}$ are positive constants and $\|v\|=\int_{\Omega}|\nabla v|^{2} \mathrm{~d} x$. Also, for any $q>1$, through Young's inequality, we obtain for the solution $u(x, t)$

$$
q\left(\frac{|\kappa(u)|}{\|\kappa(u)\|}\right)\|\kappa(u)\| \leqslant\left(\frac{c_{1}|\kappa(u)|}{\|\kappa(u)\|}\right)^{2}+\tilde{q}\|\kappa(u)\|^{2} .
$$

Hence, taking into account (4.7) we finally get

$$
\int_{\Omega} \mathrm{e}^{q \kappa(u)} \mathrm{d} x \leqslant c_{2}|\Omega| \mathrm{e}^{\tilde{q}\|\kappa(u)\|^{2}}<\infty, \quad \text { for every } t>0 .
$$

The latter implies

$$
\int_{\Omega}\left[\frac{\mathrm{e}^{\kappa(u)}}{\left(\int_{\Omega} \mathrm{e}^{\kappa(u)} \mathrm{d} x\right)^{p}}\right]^{q} \mathrm{~d} x \leqslant \frac{c_{2} \mathrm{e}^{\tilde{q}\|\kappa(u)\|^{2}}}{|\Omega|^{p q-1}}<\infty
$$

giving that

$$
g(x, t)=\frac{\mathrm{e}^{\kappa(u)}}{\left(\int_{\Omega} \mathrm{e}^{\kappa(u)} \mathrm{d} x\right)^{p}} \in L^{q}(\Omega), \quad \text { for } q>1 \text { and any } t>0 .
$$

By standard parabolic arguments $\left(L^{q}\right.$-estimates and embedding of Sobolev Theorems (see $[\mathbf{1 1}])$ ) we have that $u$ is Hölder continuous in $0 \leqslant t \leqslant T$, for every $T>0$. Finally, through Schauder-type estimates we obtain that $u$ is a uniformly bounded classical solution to problem (4.6) in $Q_{\infty}=\Omega \times[0, \infty)$.

Using the Lyapunov functional we prove some stability results concerning the steadystate problem (2.1) when $f(s)=\mathrm{e}^{\kappa(s)}$. 
Proposition 4.4. Let $f(s)=\mathrm{e}^{\kappa(s)}, p \geqslant 1$ and $\Omega=(-1,1)$. Then the solution $u(x, t)$ of problem (4.1) with $0 \leqslant u(x, 0)=\psi(x) \in L^{\infty}(\Omega) \cap H_{0}^{1}(\Omega)$ converges in $C^{1}(\bar{\Omega}) \cap C^{2}(\Omega)$ to the unique solution $w(x)$ of problem (3.1), for every $\lambda>0$.

Proof. First case. For $p>1$, according to Proposition 4.1 we have

$$
\|u(\cdot, t)\|_{C^{2+\alpha}(-1,1)} \leqslant K
$$

for some $0<\alpha \leqslant 1$ and a constant $K$ independent of $t$, hence there exists a sequence $\left(t_{n}\right)_{n \in \mathbb{N}}$ with $t_{n} \rightarrow \infty$ as $n \rightarrow \infty$, such that $\left\|u\left(\cdot, t_{n}\right)-w(\cdot)\right\|_{C^{2}(\Omega) \cap C^{1}(\bar{\Omega})} \rightarrow 0$ as $n \rightarrow \infty$ (the embedding $C^{2+\alpha}(\Omega) \rightarrow C^{2}(\Omega)$ is compact if $\Omega$ is bounded (see [1] )). Thus $\omega(\psi) \neq \emptyset$. Also, we know that $A$ is a one-element set, see Proposition 3.3 , thus it is sufficient to show that $\omega(\psi) \subseteq A$. Relation (4.5) implies that

$$
\begin{aligned}
\int_{\tau}^{t} \int_{-1}^{1} \kappa^{\prime}(u) u_{t}^{2} \mathrm{~d} x \mathrm{~d} s & =-\frac{1}{2} \int_{-1}^{1}\left\{|\nabla \kappa(u(x, t))|^{2}-|\nabla \kappa(u(x, \tau))|^{2}\right\} \mathrm{d} x \\
& \quad-\frac{\lambda}{p-1}\left\{\left(\int_{-1}^{1} \mathrm{e}^{\kappa(u(x, t))} \mathrm{d} x\right)^{1-p}-\left(\int_{-1}^{1} \mathrm{e}^{\kappa(u(x, \tau))} \mathrm{d} x\right)^{1-p}\right\} \\
& \leqslant \frac{1}{2} \int_{-1}^{1}|\nabla \kappa(u(x, \tau))|^{2} \mathrm{~d} x+\frac{\lambda}{p-1}\left(\int_{-1}^{1} \mathrm{e}^{\kappa(u(x, \tau))} \mathrm{d} x\right)^{1-p} \\
& \leqslant C,
\end{aligned}
$$

since $u \in C^{2+\alpha, 1+\alpha / 2}((-1,1) \times(0, T])$ for any $T>0$. The constant $C$ depends only on $\tau$, while it is independent of $t$. Hence $\int_{\tau}^{\infty} \int_{-1}^{1} \kappa^{\prime}(u) u_{t}^{2} \mathrm{~d} x \mathrm{~d} s \leqslant C$ and since $\kappa^{\prime}(s)$ is bounded we have

$$
\int_{\tau}^{\infty} \int_{-1}^{1} u_{t}^{2} \mathrm{~d} x \mathrm{~d} s \leqslant \tilde{C}
$$

This relation implies that there exists a sequence $\left(t_{k}\right)_{k \in \mathbb{N}}$ with $t_{k} \rightarrow \infty$ as $k \rightarrow \infty$ such that

$$
u_{t}\left(x, t_{k}\right) \rightarrow 0 \quad \text { as } t_{k} \rightarrow \infty \text { in } L^{2}((-1,1)) .
$$

In order to prove that (4.9) holds for every sequence of time we again use the fact that $u_{t}$ is bounded in $C^{\alpha, \alpha / 2}([-1,1] \times[\tau, \infty))$. This implies that $\left\|u_{t}(\cdot, t)\right\|_{L^{2}((-1,1))}=v(t)$ is uniformly continuous in $[\tau, \infty)$. Let us suppose that there exists a sequence $t_{m} \rightarrow \infty$ such that $v\left(t_{m}\right) \nrightarrow 0$ as $t_{m} \rightarrow \infty$, then there would be a constant $\sigma>0$ with $v\left(t_{m}\right) \geqslant \sigma>0$ for every $m \geqslant 1$ (redefining $\left(t_{m}\right)_{m \in \mathbb{N}}$ if it is necessary). Since $v$ is uniformly continuous we can choose $\delta>0$ such that for every $\varepsilon \in\left(0, \frac{1}{2} \sigma\right)$ we have $\left|v(t)-v\left(t_{m}\right)\right|<\varepsilon$ when $\left|t-t_{m}\right|<\delta$. Integrating over $\left(t_{m}-\delta, t_{m}+\delta\right)$ we get $\int_{t_{m}-\delta}^{t_{m}+\delta} v(t) \mathrm{d} t \geqslant(\sigma-\varepsilon) 2 \delta>\sigma \delta$ and so

$$
\int_{\tau}^{t_{m}+\delta} v(t) \mathrm{d} t \geqslant \sum_{k=1}^{m} \int_{t_{k}-\delta}^{t_{k}+\delta} v(t) \mathrm{d} t \geqslant m \sigma \delta .
$$

This relation implies that

$$
\int_{\tau}^{\infty} v(t) \mathrm{d} t=\lim _{m \rightarrow \infty} \int_{\tau}^{t_{m}+\delta} v(t) \mathrm{d} t \geqslant \lim _{m \rightarrow \infty} m \sigma \delta=\infty
$$


which contradicts $(4.8)$ (the sequence $\left(t_{n}\right)_{n \in \mathbb{N}}$ can be chosen such that $t_{m+1}-t_{m} \geqslant 2 \varepsilon$ ). Therefore,

$$
u_{t}(x, t) \rightarrow 0 \quad \text { as } t \rightarrow \infty \text { in } L^{2}((-1,1)) .
$$

Now, for every $\xi \in H_{0}^{1}((-1,1))$ we have

$$
\begin{aligned}
\int_{-1}^{1} u_{t} \xi \mathrm{d} x & =\int_{-1}^{1}\left[(\kappa(u))_{x x}+\frac{\lambda \mathrm{e}^{\kappa(u)}}{\left(\int_{-1}^{1} \mathrm{e}^{\kappa(u)} \mathrm{d} x\right)^{p}}\right] \xi \mathrm{d} x \\
& =-\int_{-1}^{1}(\kappa(u))_{x} \xi^{\prime} \mathrm{d} x+\lambda \int_{-1}^{1} \frac{\mathrm{e}^{\kappa(u)} \xi \mathrm{d} x}{\left(\int_{-1}^{1} \mathrm{e}^{\kappa(u)} \mathrm{d} x\right)^{p}}
\end{aligned}
$$

( $\xi^{\prime}$ is the generalized derivative of $\xi$ ).

Since $\omega(\psi) \neq \emptyset$, there exists a sequence $\left(t_{n}\right)_{n \in \mathbb{N}}$ with $t_{n} \rightarrow \infty$ as $n \rightarrow \infty$ such that $u\left(x, t_{n} ; \psi\right) \rightarrow w(x)$ in $C^{1}([-1,1]) \cap C^{2}((-1,1))$. Now, passing to the limit as $n \rightarrow \infty$ in (4.11), and also taking into account (4.10), we obtain that $w$ satisfies

$$
0=-\int_{-1}^{1}(\kappa(w))_{x} \xi^{\prime} \mathrm{d} x+\lambda \int_{-1}^{1} \frac{\mathrm{e}^{\kappa(w)} \xi \mathrm{d} x}{\left(\int_{-1}^{1} \mathrm{e}^{\kappa(w)} \mathrm{d} x\right)^{p}} .
$$

However, $w \in C^{1}([-1,1]) \cap C^{2}((-1,1))$ so we take

$$
0=\int_{-1}^{1}\left[(\kappa(w))_{x x}+\frac{\lambda \mathrm{e}^{\kappa(w)}}{\left(\int_{-1}^{1} \mathrm{e}^{\kappa(w)} \mathrm{d} x\right)^{p}}\right] \xi \mathrm{d} x .
$$

This relation, since $\xi$ is an arbitrary function in $H_{0}^{1}(\Omega)$, tells us that $w$ is the unique classical solution to (3.1).

Second case. In the case where $p=1$, the only thing in the proof that has changed is that $C$ now depends on $\sup _{t \geqslant \tau}\|u(\cdot, t)\|$ as well. This is because, for $p=1$, the Lyapunov functional of (4.1) has the form

$$
J[u](t)=\frac{1}{2} \int_{-1}^{1}|\nabla \kappa(u)|^{2} \mathrm{~d} x-\lambda \ln \left(\int_{-1}^{1} \mathrm{e}^{\kappa(u)} \mathrm{d} x\right) .
$$

For dimension $N=2$ the following proposition holds.

Proposition 4.5. Let $f(s)=\mathrm{e}^{\kappa(s)}$ with $\kappa(0)=0$.

(1) If $N=2, p>1$ and $\Omega=B(0,1)$, then the solution $u(r, t)$ to (4.6), where $0<$ $u(r, 0)=\psi(r) \in L^{\infty}(\Omega) \cap H_{0}^{1}(\Omega)$ is radial symmetric, converges in $C^{1}(\bar{\Omega}) \cap C^{2}(\Omega)$ to the unique solution $w(r)$ of (3.1) for every $\lambda>0$.

(2) If $N=2, p=1$ and $\Omega=B(0,1)$, then the solution $u(r, t)$ of (4.6), where $0<$ $u(r, 0)=\psi(r) \in L^{\infty}(\Omega) \cap H_{0}^{1}(\Omega)$ is radial symmetric, converges in $C^{1}(\bar{\Omega}) \cap C^{2}(\Omega)$ to the unique solution $w(r)$ of (3.1) for every $0<\lambda<8 \pi$. 
The proof follows the same steps as those of Proposition 4.4 and is therefore omitted.

Remark 4.6. If Dirichlet boundary conditions are replaced by Neumann-type boundary conditions, then again using the Lyapunov functional and provided that $u(r, 0)=$ $\psi(r)$ is radial symmetric, $u(r, t)$ is proved to be a global-in-time classical solution (see [4]).

\section{Blow-up for the exponential and $0<p<1$}

In this section we investigate under what circumstances the solutions to the problem

$$
\begin{gathered}
u_{t}=\Delta(\kappa(u))+\frac{\lambda \mathrm{e}^{\kappa(u)}}{\left(\int_{\Omega} \mathrm{e}^{\kappa(u)} \mathrm{d} x\right)^{p}}, \quad x \in \Omega, \quad t>0, \\
u(x, t)=0, \quad x \in \partial \Omega, \quad t>0 \\
u(x, 0)=\psi(x), \quad x \in \Omega
\end{gathered}
$$

( $\Omega$ is an open subset of $\mathbb{R}^{N}$ with smooth boundary $\partial \Omega$ ), blow up in finite time, when $0<p<1$. For this investigation we use energy methods, in contrast to the comparison techniques used in the case of a decreasing function $f(s)$ (see $[\mathbf{1 3}, \mathbf{1 4}])$.

By using a method due to Fila $[6,7]$, we first prove that if $u$ is a global-in-time solution to $(5.1)$, then $\kappa(u)$ should be uniformly $H_{0}^{1}(\Omega)$-bounded and afterwards we exclude the possibility of global-in-time solutions existing for $\lambda>\lambda^{*}$. In order to prove the first result we need a sequence of lemmas.

Lemma 5.1. Let $u$ be a global-in-time solution to problem (5.1), then there exists a constant $\nu=\nu(\psi, b)$ such that

$$
\int_{\Omega} K(u) \mathrm{d} x \leqslant \nu, \quad \text { for any } t \geqslant 0
$$

where $K(s)=\int_{0}^{s} \kappa(s) \mathrm{d} s$, provided that $\kappa(s) \geqslant C(K(s))^{b}, s \geqslant 0$, for some positive constants $C, b>0$ and $\int_{0}^{\infty} \kappa(s) \mathrm{d} s=\infty$.

Proof. Multiplying (5.1 a) by $\kappa(u)$ and integrating over $\Omega$ we obtain

$$
\frac{\mathrm{d}}{\mathrm{d} t} \int_{\Omega} K(u) \mathrm{d} x=-\int_{\Omega}|\nabla \kappa(u)|^{2} \mathrm{~d} x+\frac{\lambda \int_{\Omega} \kappa(u) \mathrm{e}^{\kappa(u)} \mathrm{d} x}{\left(\int_{\Omega} \mathrm{e}^{\kappa(u)} \mathrm{d} x\right)^{p}} .
$$

For some $\varepsilon \geqslant 0$, relation (5.2), through (4.5), gives

$$
\begin{aligned}
\frac{\mathrm{d}}{\mathrm{d} t} \int_{\Omega} K(u) \mathrm{d} x= & -2 J[u](t)+\frac{2 \lambda}{p-1} \frac{\int_{\Omega} \mathrm{e}^{\kappa(u)} \mathrm{d} x}{\left(\int_{\Omega} \mathrm{e}^{\kappa(u)} \mathrm{d} x\right)^{p}}+\frac{\lambda \int_{\Omega} \kappa(u) \mathrm{e}^{\kappa(u)} \mathrm{d} x}{\left(\int_{\Omega} \mathrm{e}^{\kappa(u)} \mathrm{d} x\right)^{p}} \\
\geqslant- & 2 J[\psi]+\frac{\lambda}{\left(\int_{\Omega} \mathrm{e}^{\kappa(u)} \mathrm{d} x\right)^{p}} \\
& \times\left[\int_{\Omega}\left\{\kappa(u) \mathrm{e}^{\kappa(u)}-\left(\frac{2}{1-p}+\varepsilon\right) \mathrm{e}^{\kappa(u)}\right\} \mathrm{d} x+\varepsilon \int_{\Omega} \mathrm{e}^{\kappa(u)} \mathrm{d} x\right] \\
\geqslant- & 2 J[\psi]-\lambda C_{1}|\Omega|^{1-p}+\lambda \varepsilon\left(\int_{\Omega} \mathrm{e}^{\kappa(u)} \mathrm{d} x\right)^{1-p},
\end{aligned}
$$


where the constant $C_{1}(\varepsilon)=C_{1}>0$ is chosen such that

$$
\kappa(u) \mathrm{e}^{\kappa(u)}-\left(\frac{2}{1-p}+\varepsilon\right) \mathrm{e}^{\kappa(u)} \geqslant-C_{1}
$$

(such a choice is always possible since $\kappa(s) \rightarrow \infty$ as $s \rightarrow \infty$ ).

Taking into account our hypothesis $\kappa(s) \geqslant C(K(s))^{b}$ for $s \geqslant 0$ and applying first Hölder's and then Jensen's inequality we get

$$
\begin{aligned}
\left(\int_{\Omega} \mathrm{e}^{\kappa(u)} \mathrm{d} x\right)^{1-p} & \geqslant\left(\int_{\Omega} \mathrm{e}^{C(K(u))^{b}} \mathrm{~d} x\right)^{1-p} \\
& \geqslant C_{3}\left(\int_{\Omega} K(u)^{4 /(1-p)} \mathrm{d} x\right)^{1-p} \\
& \geqslant C_{4}\left(\int_{\Omega} K^{4}(u) \mathrm{d} x\right) \\
& \geqslant C_{4}\left(\int_{\Omega} K(u) \mathrm{d} x\right)^{4}
\end{aligned}
$$

Hence

$$
\frac{\mathrm{d}}{\mathrm{d} t} \int_{\Omega} K(u) \mathrm{d} x \geqslant \lambda C_{4} \varepsilon\left(\int_{\Omega} K(u) \mathrm{d} x\right)^{4}-C_{2},
$$

where $C_{2}=2 J[\psi]+\lambda C_{1}|\Omega|^{1-p}$.

Suppose there exists $t_{0}$ such that

$$
\Lambda\left(t_{0}\right)=\int_{\Omega} K\left(u\left(x, t_{0}\right)\right) \mathrm{d} x>\left(\frac{C_{2}}{\lambda C_{4} \varepsilon}\right)^{1 / 4}=\nu(b, \psi),
$$

then from (5.3) we deduce that $\Lambda(t)$ is an increasing function for $t \geqslant t_{0}$ and there exists $\delta>0$ such that $\Lambda^{\prime}(t) \geqslant \delta \Lambda^{4}(t)$, for $t \geqslant t_{0}$. Integrating the latter inequality for $t \geqslant t_{0}$ we obtain

$$
t \leqslant \frac{1}{\delta} \int_{\Lambda\left(t_{0}\right)}^{\Lambda(t)} \frac{\mathrm{d} s}{s^{4}}+t_{0}<\frac{1}{\delta} \int_{\Lambda\left(t_{0}\right)}^{\infty} \frac{\mathrm{d} s}{s^{4}}+t_{0}<\infty,
$$

which implies the existence of a $t^{*}<\infty$ such that $\Lambda(t) \rightarrow \infty$ as $t \rightarrow t^{*}-$. Therefore, since $K(s) \rightarrow \infty$ only if $s \rightarrow \infty$, we obtain that $u(x, t)$ blows up in finite time $T^{*}<t^{*}<\infty$ as well, i.e. $\|u(\cdot, t)\| \rightarrow \infty$ as $t \rightarrow T^{*}-$, which contradicts the fact that $u$ is a global-in-time solution.

Lemma 5.1 is essential in proving that the solution $u$ to (5.1) blows up for 'large enough' initial data.

Proposition 5.2. Let $\kappa(u)=u^{m}, m>1$, then for every $\psi>0$ with $\kappa(\psi)=\psi^{m} \in$ $H_{0}^{1}(\Omega)$ there exists a $\mu^{*}$ such that for every $\mu>\mu^{*}$ the solution $u$ to (5.1) with $u(x, 0)=$ $\mu \psi(x)$ blows up in finite time. 
Proof. We set

$$
\pi(\mu):=J[\mu \psi]=\frac{1}{2} \int_{\Omega}\left|\nabla(\mu \psi)^{m}\right|^{2} \mathrm{~d} x-\frac{\lambda}{1-p}\left(\int_{\Omega} \mathrm{e}^{\mu^{m} \psi^{m}} \mathrm{~d} x\right)^{1-p} .
$$

Suppose that $C$ is a constant such that $\mathrm{e}^{\mu^{m}} \psi^{m}>C\left(\mu^{m} \psi^{m}\right)^{(2+\varepsilon) /(1-p)}$, then

$$
\pi(\mu)<\frac{1}{2} \mu^{2 m} \int_{\Omega}\left|\nabla \psi^{m}\right|^{2} \mathrm{~d} x-\frac{\lambda C^{1-p}}{1-p} \mu^{(2+\varepsilon) m}\left(\int_{\Omega} \psi^{((2+\varepsilon) m) /(1-p)} \mathrm{d} x\right)^{1-p} .
$$

The latter implies that $\pi(\mu) \rightarrow-\infty$ as $\mu \rightarrow \infty$. Therefore, there exists $\mu_{0}$ such that $\pi(\mu)<0$ for $\mu \geqslant \mu_{0}$. Following the same lines as in the proof of Lemma 5.1 we obtain

$$
\frac{1}{m+1} \frac{\mathrm{d}}{\mathrm{d} t} \int_{\Omega} u^{m+1} \mathrm{~d} x \geqslant \lambda \varepsilon C_{4}\left(\int_{\Omega} u^{m+1} \mathrm{~d} x\right)^{4}-C_{2},
$$

where $C_{2}=2 J[\mu \psi]+\lambda C_{1}|\Omega|^{1-p}$.

Now, by choosing

$$
\mu^{*}=\max \left\{\mu_{0},\left[\left(\frac{C_{2}^{*}}{\lambda \varepsilon C_{4}}\right)^{1 / 4}\|\psi\|_{m+1}^{-m-1}\right]^{1 /(m+1)}\right\},
$$

where $C_{2}^{*}=\lambda C_{1}|\Omega|^{1-p}$, we get $\Lambda(0)=\int_{\Omega}(\mu \psi)^{m+1} \mathrm{~d} x>\left(C_{2} / \lambda \varepsilon C_{4}\right)$, and due to (5.4) we obtain that $\Lambda(t)=\int_{\Omega} u^{m+1}(x, t) \mathrm{d} x$ is an increasing function for $t>0$ and more precisely there exists $\delta>0$ such that $\Lambda^{\prime}(t) \geqslant \delta \Lambda^{4}(t)$ for $t>0$. The latter implies that there exists a $t^{*}<\infty$ such that $\Lambda(t) \rightarrow \infty$ as $t \rightarrow t^{*}-$, which implies that $u$ blows up in finite time $T^{*} \leqslant t^{*}$.

Remark 5.3. Proposition 5.2 is still valid if $\kappa(s)$ is a homogeneous function, i.e. if there exist $\mu, \alpha>0$ such that $\kappa(\mu s)=\mu^{\alpha} \kappa(s)$.

Remark 5.4. Proposition 5.2 is analogous to that which holds for the local reactiondiffusion problem (see $[\mathbf{1 0}]$ ). This is not something unexpected, since for $0<p<1$ the contribution of the denominator of the non-local term to the problem (5.1) is quite small.

Lemma 5.5. Let $\kappa(s)$ satisfy the hypotheses of Lemma 5.1. If $\int_{\Omega}|\nabla \kappa(u)|^{2} \mathrm{~d} x \rightarrow \infty$ as $t \rightarrow t_{\max }$, where $t_{\max }$ is the maximal existence time for the solution $u$ to problem (5.1), then $t_{\max }<\infty$.

Proof. Suppose that $t_{\max }=\infty$, then $u$ is a global-in-time solution. Defining $E(t)=\int_{0}^{t} \int_{\Omega} K(u(x, s)) \mathrm{d} x \mathrm{~d} s$, we have

$$
\begin{aligned}
E^{\prime}(t) & =\int_{\Omega} K(u(x, t)) \mathrm{d} x \\
& =\int_{\Omega} K(\psi(x)) \mathrm{d} x+\int_{0}^{t} \int_{\Omega}(K(u(x, s)))_{t} \mathrm{~d} x \mathrm{~d} s \\
& =\int_{\Omega} K(\psi) \mathrm{d} x+\int_{0}^{t}\left(-\int_{\Omega}|\nabla \kappa(u)|^{2} \mathrm{~d} x+\lambda \int_{\Omega} \frac{\kappa(u) \mathrm{e}^{\kappa(u)}}{\left(\int_{\Omega} \mathrm{e}^{\kappa(u)} \mathrm{d} x\right)^{p}} \mathrm{~d} x\right) \mathrm{d} s
\end{aligned}
$$


and using (4.5) we obtain

$$
\begin{aligned}
E^{\prime \prime}(t)=-\int_{\Omega}|\nabla \kappa(u)|^{2} \mathrm{~d} x+ & \lambda \int_{\Omega} \frac{\kappa(u) \mathrm{e}^{\kappa(u)}}{\left(\int_{\Omega} \mathrm{e}^{\kappa(u)} \mathrm{d} x\right)^{p}} \mathrm{~d} x \\
=-(2+\varepsilon) J[u](t)+\frac{1}{2} \varepsilon \int_{\Omega}|\nabla \kappa(u)|^{2} \mathrm{~d} x & \quad \lambda \int_{\Omega} \frac{\kappa(u) \mathrm{e}^{\kappa(u)}}{\left(\int_{\Omega} \mathrm{e}^{\kappa(u)} \mathrm{d} x\right)^{p}} \mathrm{~d} x+\frac{(2+\varepsilon) \lambda}{p-1}\left(\int_{\Omega} \mathrm{e}^{\kappa(u)} \mathrm{d} x\right)^{1-p} \\
\geqslant-(2+\varepsilon) J[\psi]+\frac{1}{2} \varepsilon \int_{\Omega}|\nabla \kappa(u)|^{2} \mathrm{~d} x & \quad \frac{\lambda \int_{\Omega} \kappa(u) \mathrm{e}^{\kappa(u)} \mathrm{d} x}{\left(\int_{\Omega} \mathrm{e}^{\kappa(u)} \mathrm{d} x\right)^{p}}-\frac{\lambda(2+\varepsilon)}{1-p} \frac{\int_{\Omega} \mathrm{e}^{\kappa(u)} \mathrm{d} x}{\left(\int_{\Omega} \mathrm{e}^{\kappa(u)} \mathrm{d} x\right)^{p}} .
\end{aligned}
$$

However,

$$
\begin{aligned}
\frac{\lambda \int_{\Omega} \kappa(u) \mathrm{e}^{\kappa(u)} \mathrm{d} x}{\left(\int_{\Omega} \mathrm{e}^{\kappa(u)} \mathrm{d} x\right)^{p}}-\frac{\lambda(2+\varepsilon)}{1-p} \frac{\int_{\Omega} \mathrm{e}^{\kappa(u)} \mathrm{d} x}{\left(\int_{\Omega} \mathrm{e}^{\kappa(u)} \mathrm{d} x\right)^{p}} & \\
= & \frac{\lambda}{\left(\int_{\Omega} \mathrm{e}^{\kappa(u)} \mathrm{d} x\right)^{p}} \int_{\Omega}\left\{\kappa(u) \mathrm{e}^{\kappa(u)}-\frac{2+\varepsilon}{1-p} \mathrm{e}^{\kappa(u)}\right\} \mathrm{d} x \geqslant-\frac{\lambda C_{2}}{|\Omega|^{p-1}} \equiv-C_{3},
\end{aligned}
$$

where $C_{2}(\varepsilon)=C_{2}$ is a constant such that $\kappa(u) \mathrm{e}^{\kappa(u)}-((2+\varepsilon) /(1-p)) \mathrm{e}^{\kappa(u)}>-C_{2}$. Therefore, we take

$$
E^{\prime \prime}(t) \geqslant \frac{1}{2} \varepsilon \int_{\Omega}|\nabla \kappa(u)|^{2} \mathrm{~d} x-(2+\varepsilon) J[\psi]-C_{3},
$$

and since $\varepsilon>0, \int_{\Omega}|\nabla \kappa(u)|^{2} \mathrm{~d} x \rightarrow \infty$ as $t \rightarrow \infty$, we get $E^{\prime \prime}(t) \rightarrow \infty$ as $t \rightarrow \infty$. The latter implies that for every $N>0$ there exists $t_{0}=t_{0}(N)$ such that $E^{\prime \prime}(t)>N$ for $t>t_{0}$, so $E^{\prime}(t)>N t+N_{1}$ for $t>t_{0}\left(N_{1}=N t_{0}+E^{\prime}\left(t_{0}\right)\right)$ and hence $E^{\prime}(t) \rightarrow \infty$ as $t \rightarrow \infty$, which contradicts Lemma 5.1.

Lemma 5.6. Let $u(x, t ; \psi)$ be a global-in-time solution to problem (5.1) with $\omega(\psi) \neq \emptyset$. If $w \in \omega(\psi)$ is a steady-state solution to problem (5.1), then $\int_{\Omega}|\nabla \kappa(w)|^{2} \mathrm{~d} x \leqslant M=$ $M(\psi)$.

Proof. Since $J[u]$ is a Lyapunov functional, the function $t \rightarrow J[u](t)$ is decreasing for $t>0$, therefore $J[w]=\inf _{t>0} J[u](t) \leqslant J[\psi]$ for every $w \in \omega(\psi)$, and so $(2+\varepsilon) J[w] \leqslant$ $(2+\varepsilon) J[\psi]$. However,

$$
\begin{aligned}
(2+\varepsilon) J[w] & =(2+\varepsilon)\left[\frac{1}{2} \int_{\Omega}|\nabla \kappa(w)|^{2} \mathrm{~d} x+\frac{\lambda}{p-1}\left(\int_{\Omega} \mathrm{e}^{\kappa(w)} \mathrm{d} x\right)^{1-p}\right] \\
& =\frac{1}{2} \varepsilon \int_{\Omega}|\nabla \kappa(w)|^{2} \mathrm{~d} x+\int_{\Omega}|\nabla \kappa(w)|^{2} \mathrm{~d} x+\frac{\lambda(2+\varepsilon)}{p-1}\left(\int_{\Omega} \mathrm{e}^{\kappa(w)} \mathrm{d} x\right)^{1-p},
\end{aligned}
$$


but $w$ is a steady-state solution so we have

$$
\int_{\Omega}|\nabla \kappa(w)|^{2} \mathrm{~d} x=\lambda \int_{\Omega} \frac{\kappa(w) \mathrm{e}^{\kappa(w)}}{\left(\int_{\Omega} \mathrm{e}^{\kappa(w)} \mathrm{d} x\right)^{p}} \mathrm{~d} x
$$

Hence the following expression holds:

$$
\begin{aligned}
(2+\varepsilon) J[\psi] & \geqslant \frac{1}{2} \varepsilon \int_{\Omega}|\nabla \kappa(w)|^{2} \mathrm{~d} x+\frac{\lambda \int_{\Omega}\left\{\kappa(w) \mathrm{e}^{\kappa(w)}-((2+\varepsilon) /(1-p)) \mathrm{e}^{\kappa(w)}\right\} \mathrm{d} x}{\left(\int_{\Omega} \mathrm{e}^{\kappa(w)} \mathrm{d} x\right)^{p}} \\
& \geqslant \frac{1}{2} \varepsilon \int_{\Omega}|\nabla \kappa(w)|^{2} \mathrm{~d} x-\frac{\lambda C}{|\Omega|^{p-1}},
\end{aligned}
$$

where $C=C(\varepsilon)>0$ is a constant such that

$$
\int_{\Omega}\left\{\kappa(w) \mathrm{e}^{\kappa(w)}-\frac{(2+\varepsilon)}{1-p} \mathrm{e}^{\kappa(w)}\right\} \mathrm{d} x \geqslant-C .
$$

Finally, we obtain

$$
\int_{\Omega}|\nabla \kappa(w)|^{2} \mathrm{~d} x \leqslant\left(2+\frac{4}{\varepsilon}\right) J[\psi]+\frac{2}{\varepsilon} \frac{\lambda C}{|\Omega|^{p}}=M(\psi),
$$

for any steady-state solution $w \in \omega(\psi)$.

Now, we introduce a functional $\Pi(t) \equiv \Pi[u](t)=\int_{\Omega} \mathrm{e}^{\kappa(u(x, t))} \mathrm{d} x$, which will be used to exclude the possibility

$$
\lim _{t \rightarrow \infty} \sup \int_{\Omega}|\nabla \kappa(u(x, t))|^{2} \mathrm{~d} x=\infty \quad \text { and } \quad \lim _{t \rightarrow \infty} \inf \int_{\Omega}|\nabla \kappa(u(x, t))|^{2} \mathrm{~d} x<\infty .
$$

The functional $\Pi(t)$ satisfies a result analogous to Theorem 3.1 in $[\mathbf{1 2}]$.

Lemma 5.7. Let $\Omega=(-1,1)$ and $\kappa(s) \rightarrow \infty$ as $s \rightarrow \infty$. If $\tau>\sigma$ and $\beta, B, \gamma$ are positive constants with $B>\gamma$, satisfying

$$
\begin{gathered}
\Pi(\tau)=B^{2}, \quad \Pi(\sigma)=\gamma^{2}, \quad \Pi(t) \leqslant B^{2}, \quad \text { for } t \in[\sigma, \tau] \\
H(\sigma)=\int_{\Omega}|\nabla \kappa(u(x, \sigma))|^{2} \mathrm{~d} x \leqslant \beta^{2}
\end{gathered}
$$

then there exists a positive constant $\delta=\delta\left(B^{2}-\gamma^{2}, \lambda, p\right)$ such that $\tau-\sigma \geqslant \delta$.

Proof. From $(4.5)$ we take $J[u](t) \leqslant J[u](\sigma)$ for $t \in[\sigma, \tau]$ and, since $p<1, \frac{1}{2} H(t) \leqslant$ $\frac{1}{2} H(\sigma)+(\lambda /(1-p)) \Pi(t)^{1-p}$ holds. Due to $(5.6)$, (5.7) the previous relation becomes $\frac{1}{2} H(t) \leqslant \frac{1}{2} \beta^{2}+(\lambda /(1-p)) B^{2(1-p)}$. If, now, without loss of generality, we assume that $B>1$, we have

$$
H(t) \leqslant \frac{2}{1-p}\left(\beta^{2}+\lambda B^{2}\right) \leqslant\left[\frac{2}{1-p}(\beta+\lambda B)\right]^{2}, \quad \text { for } t \in[\sigma, \tau] .
$$


Relation (5.8), through the one-dimensional Sobolev inequality (see [1] ), gives

$$
\kappa(u(x, t)) \leqslant \frac{2 C}{1-p}(\beta+\lambda B) \equiv C_{1}, \quad \text { for }(x, t) \in[-1,1] \times[\sigma, \tau] .
$$

Evaluating $\Pi[u](t)$ upon a solution $u$ of (5.1), differentiating afterwards with respect to $t$ and using (5.9) we obtain

$$
\begin{aligned}
\Pi^{\prime}(t) & =\int_{-1}^{1} \kappa^{\prime}(u) \mathrm{e}^{\kappa(u)} u_{t} \mathrm{~d} x \\
& =\int_{-1}^{1} \kappa^{\prime}(u) \mathrm{e}^{\kappa(u)}(\kappa(u))_{x x} \mathrm{~d} x+\lambda \int_{-1}^{1} \frac{\kappa^{\prime}(u) \mathrm{e}^{2 \kappa(u)}}{\left(\int_{-1}^{1} \mathrm{e}^{\kappa(u)} \mathrm{d} x\right)^{p}} \mathrm{~d} x \\
& \leqslant \int_{-1}^{1} \kappa^{\prime}(u) \mathrm{e}^{\kappa(u)}(\kappa(u))_{x x} \mathrm{~d} x+\lambda \kappa^{\prime}\left(\kappa^{-1}\left(C_{1}\right) \mathrm{e}^{C_{1}}\left(\int_{-1}^{1} \mathrm{e}^{\kappa(u)} \mathrm{d} x\right)^{1-p} .\right.
\end{aligned}
$$

For the first term of (5.10) we have

$$
\int_{-1}^{1} \kappa^{\prime}(u) \mathrm{e}^{\kappa(u)}(\kappa(u))_{x x} \mathrm{~d} x \leqslant-\int_{-1}^{1}\left[\kappa^{\prime}(u)+\kappa^{\prime \prime}(u)\right] \mathrm{e}^{\kappa(u)} \kappa^{\prime}(u) u_{x}^{2} \mathrm{~d} x \leqslant 0,
$$

since $u_{x}(-1, t)>0, u_{x}(1, t)<0$ and $\kappa^{\prime}(s) \geqslant 0, \kappa^{\prime \prime}(s) \geqslant 0$ for $s \geqslant 0$. Hence we get $\Pi^{\prime}(t) \leqslant \lambda \kappa^{\prime}\left(\kappa^{-1}\left(C_{1}\right)\right) 2^{1-p} \mathrm{e}^{(2-p) C_{1}}$ for $t \in[\tau, \sigma]$, implying

$$
B^{2}-\gamma^{2}=\Pi(\tau)-\Pi(\sigma) \leqslant(\tau-\sigma) \lambda \kappa^{\prime}\left(\kappa^{-1}\left(C_{1}\right)\right) 2^{1-p} \mathrm{e}^{(2-p) C_{1}} .
$$

The latter gives $\delta=\left(B^{2}-\gamma^{2}\right) /\left(\lambda \kappa^{\prime}\left(\kappa^{-1}\left(C_{1}\right)\right) 2^{1-p} \mathrm{e}^{(2-p) C_{1}}\right)$.

Lemma 5.8. Let $\Omega=(-1,1)$ and $\kappa(s) \rightarrow \infty$ as $s \rightarrow \infty$. If $u(x, t ; \psi)$ is a global-in-time solution to problem (5.1) with

$$
\lim _{t \rightarrow \infty} \inf \|\kappa(u(\cdot, t))\|_{H_{0}^{1}(\Omega)}<+\infty \quad \text { and } \quad \lim _{t \rightarrow \infty} \sup \|\kappa(u(\cdot, t))\|_{H_{0}^{1}(\Omega)}=+\infty,
$$

then for every large enough constant $B$ there exists a steady-state solution $w \in \omega(\psi)$ such that $\Pi[w]=B^{2}$.

Proof. Below we use the norm

$$
\|\kappa(u(\cdot, t))\|_{H_{0}^{1}(\Omega)}=\left(\int_{\Omega}|\nabla \kappa(u)|^{2} \mathrm{~d} x\right)^{1 / 2}
$$

instead of the usual norm of $H_{0}^{1}(\Omega)$. From the one-dimensional Sobolev inequality and (5.11) we get

$$
\lim _{t \rightarrow \infty} \inf \Pi(t)=\gamma^{2}<+\infty
$$

Since also

$$
\frac{1}{2} \int_{\Omega}|\nabla \kappa(u(x, t))|^{2} \mathrm{~d} x \leqslant \frac{1}{2} \int_{\Omega}|\nabla \kappa(\psi)|^{2} \mathrm{~d} x+\frac{\lambda}{1-p} \Pi^{1-p}(t),
$$


from our hypothesis (5.11) we obtain

$$
\lim _{t \rightarrow \infty} \sup \Pi(t)=+\infty
$$

Now, due to (5.12) and (5.14) we can find two sequences $\left(\tau_{m}\right)_{m \in \mathbb{N}},\left(\sigma_{m}\right)_{m \in \mathbb{N}}$ diverging to infinity and satisfying the relations $\Pi\left(\tau_{m}\right)=B^{2}, \Pi\left(\sigma_{m}\right)=\gamma^{2}, \Pi(t) \leqslant B^{2}$ for $t \in$ $\left[\sigma_{m}, \tau_{m}\right]$ and any arbitrary constant $B \in\left(\liminf _{t \rightarrow \infty} \Pi(t), \lim _{\sup _{t \rightarrow \infty}} \Pi(t)\right)(\operatorname{see}[\mathbf{6}, \mathbf{1 2}])$. Furthermore, relation (5.13) for $t=\sigma_{m}$ gives $H\left(\sigma_{m}\right) \leqslant(2 /(1-p))\left(H(0)+\lambda \gamma^{2(1-p)}\right)$. Applying Lemma 5.7 for $\beta=(2 /(1-p))\left(H(0)+\lambda \gamma^{2(1-p)}\right)$ we take a constant $\delta>0$ satisfying $\tau_{m}-\sigma_{m}>\delta$. Now, relation (4.5) with $t=\tau_{m}$ gives, sending $m$ to infinity, $M(u)_{t} \in L^{2}((-1,1) \times(0, \infty))$ (where $\left.M^{\prime}(s)=\left(\kappa^{\prime}(s)\right)^{1 / 2}\right)$, and therefore

$$
\int_{\tau_{m}-\delta}^{\tau_{m}} \int_{-1}^{1}\left(M_{t}(u)\right)^{2} \mathrm{~d} x \mathrm{~d} t \rightarrow 0 \quad \text { as } m \rightarrow \infty .
$$

We have, using standard parabolic arguments (see [11, Theorem V.3.1]), that the sequence $\left(u_{m}(\cdot, t)\right)_{m \in \mathbb{N}}$ (where $\left.u_{m}(x, t)=u\left(x, \tau_{m}-\delta+t\right)\right)$ is uniformly bounded with respect to $m$ in $C^{1+\alpha}((-1,1))$, for some $0<\alpha<1$, and hence $\left(u_{m}(\cdot, t)\right)_{m \in \mathbb{N}}$ is precompact in $H^{1}(\Omega)$. Therefore, there exists a subsequence $\left(u_{m_{\kappa}}(\cdot, t)\right)_{\kappa \in \mathbb{N}}$ converging to a limit function $w \in \omega(\psi)$ with respect to the $H_{0}^{1}(\Omega)$-norm. We claim that the limit function $w$ is a steady-state solution. Indeed, due to $(5.15)$ we have

$$
\int_{-1}^{1}\left|M\left(u_{m}\left(x, t_{2}\right)\right)-M\left(u_{m}\left(x, t_{1}\right)\right)\right|^{2} \mathrm{~d} x \leqslant \delta \int_{-1}^{1} \int_{0}^{\delta}\left|M_{t}\left(u_{m}\right)\right|^{2} \mathrm{~d} t \mathrm{~d} x \rightarrow 0, \quad m \rightarrow \infty,
$$

for $0<t_{1}<t_{2}<\delta$. In other words, we assume that $M(w)$ is independent of $t$, and so $w$ is as well. Hence there holds

$$
\begin{aligned}
\delta \int_{-1}^{1}\left[(\kappa(w(x)))_{x} \phi_{x}(x)-\frac{\lambda \mathrm{e}^{\kappa(w(x))} \phi(x)}{\left(\int_{-1}^{1} \mathrm{e}^{\kappa(w(x))} \mathrm{d} x\right)^{p}}\right] \mathrm{d} x \\
=\int_{0}^{\delta} \int_{-1}^{1}\left[(\kappa(w(x)))_{x} \phi_{x}(x)-\frac{\lambda \mathrm{e}^{\kappa(w(x))} \phi(x)}{\left(\int_{-1}^{1} \mathrm{e}^{\kappa(w(x))} \mathrm{d} x\right)^{p}}\right] \mathrm{d} x \mathrm{~d} t \\
=\lim _{m \rightarrow \infty} \int_{0}^{\delta} \int_{-1}^{1}\left[\left(\kappa\left(u_{m}(x, t)\right)\right)_{x} \phi_{x}(x)-\frac{\lambda \mathrm{e}^{\kappa\left(u_{m}(x, t)\right)} \phi(x)}{\left(\int_{-1}^{1} \mathrm{e}^{\kappa\left(u_{m}(x, t)\right)} \mathrm{d} x\right)^{p}}\right] \mathrm{d} x \mathrm{~d} t \\
=-\lim _{m \rightarrow \infty} \int_{0}^{\delta} \int_{-1}^{1}\left[\left(\kappa\left(u_{m}(x, t)\right)\right)_{x x}+\frac{\lambda \mathrm{e}^{\kappa\left(u_{m}(x, t)\right)}}{\left(\int_{-1}^{1} \mathrm{e}^{\kappa\left(u_{m}(x, t)\right)} \mathrm{d} x\right)^{p}}\right] \phi(x) \mathrm{d} x \mathrm{~d} t \\
=-\lim _{m \rightarrow \infty} \int_{-1}^{1}\left(\int_{0}^{\delta} u_{t}\left(x, \tau_{m}-\delta+t\right) \mathrm{d} t\right) \phi(x) \mathrm{d} x \\
=-\lim _{m \rightarrow \infty} \int_{-1}^{1}\left[u\left(x, \tau_{m}\right)-u\left(x, \tau_{m}-\delta\right)\right] \phi(x) \mathrm{d} x=0,
\end{aligned}
$$


for every $\phi \in H_{0}^{1}((-1,1))$. Since $w$ is smooth, we finally take that

$$
\int_{-1}^{1}\left[(\kappa(w))_{x x}+\frac{\lambda \mathrm{e}^{\kappa(w)}}{\left(\int_{-1}^{1} \mathrm{e}^{\kappa(w)} \mathrm{d} x\right)^{p}}\right] \phi(x) \mathrm{d} x=0, \quad \text { for every } \phi \in H_{0}^{1}((-1,1)),
$$

therefore $w$ is a steady-state solution to (5.1) satisfying $\Pi[w]=\int_{-1}^{1} \mathrm{e}^{\kappa(w)} \mathrm{d} x=B^{2}$.

Now it can be proved that the global-in-time solutions of (5.1) are uniformly bounded with respect to $t>0$. More precisely, the following proposition holds.

Proposition 5.9. Let $\Omega=(-1,1), \psi \in H_{0}^{1}(\Omega)$ and $\kappa(s)$ be a function such that Lemmas 5.7 and 5.8 are valid. If $u(x, t ; \psi)$ is a global-in-time solution to (5.1), then

(i) $\sup _{t \geqslant 0}\|\kappa(u(\cdot, t))\|_{H_{0}^{1}(\Omega)}<\infty$, and

(ii) $\sup _{t \geqslant \tau}\|u(\cdot, t)\|_{\infty}<\infty$ for any $\tau>0$.

Proof. Suppose that (i) is not true, then due to Lemma 5.1 we take

$$
\|\kappa(u(\cdot, t))\|_{H_{0}^{1}(\Omega)} \nrightarrow \infty \quad \text { as } t \rightarrow \infty
$$

and so

$$
\lim _{t \rightarrow \infty} \sup \|\kappa(u(\cdot, t))\|_{H_{0}^{1}(\Omega)}=+\infty \quad \text { and } \quad \lim _{t \rightarrow \infty} \inf \|\kappa(u(\cdot, t))\|_{H_{0}^{1}(\Omega)}<\infty .
$$

Then Lemma 5.8 implies that for any large enough constant $B$, there exists $w \in \omega(\psi)$ satisfying

$$
\int_{-1}^{1} \mathrm{e}^{\kappa(w)} \mathrm{d} x=\mathrm{e}^{\kappa(w(\xi))}=B^{2}, \quad \text { for some } \xi \in(-1,1) .
$$

From the one-dimensional Sobolev inequality we get

$$
\left(\int_{-1}^{1}|\nabla \kappa(w)|^{2} \mathrm{~d} x\right)^{1 / 2} \geqslant C\|w\|_{\infty} \geqslant C \kappa(w(x)), \quad \text { for every } x \in[-1,1],
$$

and (5.16) leads to $\int_{-1}^{1}|\nabla \kappa(w)|^{2} \mathrm{~d} x \geqslant\left(C \ln B^{2}\right)^{2}$. According to Lemma 5.6 there exists a constant $M=M(\psi)$ such that $\int_{-1}^{1}|\nabla \kappa(w)|^{2} \mathrm{~d} x \leqslant M$. The two previous relations imply that $B \leqslant\left(\mathrm{e}^{\sqrt{\Lambda} / C}\right)^{1 / 2}$, which contradicts the fact that $B$ is an arbitrary large constant. This completes the proof of (i).

From (i) and the one-dimensional Sobolev inequality we take a constant $C_{1}$ such that $\sup _{t \geqslant \tau}\|\kappa(u(\cdot, t))\|_{\infty} \leqslant C_{1}$ for every $\tau>0$. Since $\kappa(s) \rightarrow \infty$ only if $s \rightarrow \infty$ we get that $u(x, t)$ is uniformly bounded with respect to $t$, i.e. $\sup _{t \geqslant \tau}\|u(\cdot, t)\|_{\infty}<\infty$ for any $\tau>0$.

For the two-dimensional case $N=2$, we will prove a result analogous to Theorem 5.9. Due to the nature of the two-dimensional Sobolev inequality we should modify the proof of Lemma 5.7, which is the key result for the proof of Lemma 5.8. In this case the Gilbarg-Trudinger inequality will play the role of the Sobolev inequality (see [12]). 
Lemma 5.10. Let $N=2, \Omega$ be a bounded subset of $\mathbb{R}^{2}$ and $\kappa(s) \rightarrow \infty$ as $s \rightarrow \infty$. If $\tau>\sigma$ and $\beta, B, \gamma$ are positive constants with $B>\gamma$, satisfying $\Pi(\tau)=B^{2}, \Pi(\sigma)=\gamma^{2}$, $\Pi(t) \leqslant B^{2}$ for $t \in[\sigma, \tau]$ and $H(\sigma)=\int_{\Omega}|\nabla \kappa(u(x, \sigma))|^{2} \mathrm{~d} x \leqslant \beta^{2}$, then there exists a positive constant $\delta=\delta\left(B^{2}-\gamma^{2}, \lambda, p\right)$ such that $\tau-\sigma \geqslant \delta$.

Proof. Due to the hypothesis, as in the proof of Lemma 5.7 , we get $H(t) \leqslant[(2 /(1-$ $p))(\beta+\lambda B)]^{2} \equiv C$ for $t \in[\sigma, \tau]$. The latter, through the Gilbarg-Trudinger inequality, gives

$$
\int_{\Omega} \mathrm{e}^{q \kappa(u)} \mathrm{d} x \leqslant C_{2}|\Omega| \mathrm{e}^{\tilde{q} H(t)} \leqslant C_{2}|\Omega| \mathrm{e}^{C_{1}}, \quad \text { for } t \in[\sigma, \tau],
$$

where $C_{1}, C_{2}$ are positive constants, with $C_{1}=\tilde{q}[(2 /(1-p))(\beta+\lambda B)]^{2}$ and $q>1$. Hence the following relation holds:

$$
\int_{\Omega}\left[\frac{\mathrm{e}^{\kappa(u)}}{\left(\int_{\Omega} \mathrm{e}^{\kappa(u)} \mathrm{d} x\right)^{p}}\right]^{q} \mathrm{~d} x \leqslant \frac{C_{2} \mathrm{e}^{C_{1}}}{|\Omega|^{p q-1}}<\infty, \quad \text { for } t \in[\sigma, \tau] .
$$

Using now-standard parabolic arguments we get that the solution $u$ to (5.1) is bounded in $\bar{\Omega} \times[\sigma, \tau]$ by a constant $C=C(B, \lambda)$. Following the same lines as in the proof of Lemma 5.7 we take (since $\partial u / \partial n<0$ in $\partial \Omega$ )

$$
\begin{aligned}
\Pi^{\prime}(t) & \leqslant \int_{\partial \Omega} \kappa^{\prime 2}(u) \mathrm{e}^{\kappa(u)} \frac{\partial u}{\partial n} \mathrm{~d} S-\int_{\Omega}\left[\kappa^{\prime}(u)+\kappa^{\prime \prime}(u)\right] \mathrm{e}^{\kappa(u)} \kappa^{\prime}(u)|\nabla u|^{2} \mathrm{~d} x \\
+\lambda \kappa^{\prime}(C) \mid S & \\
& \leqslant \lambda \kappa^{\prime}(C)|\Omega|^{1-p} \mathrm{e}^{(2-p) \kappa(C)} \\
& \equiv \tilde{C}
\end{aligned}
$$

Integrating the latter over $[\sigma, \tau]$ we obtain

$$
B^{2}-\gamma^{2} \leqslant(\tau-\sigma) \tilde{C} \text { and hence } \delta=\left(B^{2}-\gamma^{2}\right) / \tilde{C}
$$

In the case where $N=2$, the only thing that should be changed in the proof of Lemma 5.8 is that we now use the Gilbarg-Trudinger inequality instead of the one-dimensional Sobolev inequality to prove that $\lim _{t \rightarrow \infty}$ inf $\Pi(t)<+\infty$ when $\lim _{t \rightarrow \infty}$ inf $H(t)<+\infty$. Therefore, the following lemma holds.

Lemma 5.11. Let $N=2, \Omega$ be a bounded subset of $\mathbb{R}^{2}$ and $\kappa(s) \rightarrow \infty$ as $s \rightarrow \infty$. If $u(x, t ; \psi)$ is a global-in-time solution of (5.1) with

$$
\lim _{t \rightarrow \infty} \inf \|\kappa(u(\cdot, t))\|_{H_{0}^{1}(\Omega)}<+\infty \quad \text { and } \quad \lim _{t \rightarrow \infty} \sup \|\kappa(u(\cdot, t))\|_{H_{0}^{1}(\Omega)}=+\infty,
$$

then for every arbitrary large constant $B$ there exists a steady-state solution $w \in \omega(\psi)$ satisfying $\Pi[w]=B^{2}$.

An immediate consequence of the two previous lemmas is the following result. 
Proposition 5.12. Let $\Omega$ be a bounded subset of $\mathbb{R}^{2}, \psi \in H_{0}^{1}(\Omega)$ and let $\kappa(s)$ be a function such that Lemmas 5.10 and 5.11 are valid. If $u(x, t ; \psi)$ is a global-in-time solution of (5.1), then

(i) $\sup _{t \geqslant 0}\|\kappa(u(\cdot, t))\|_{H_{0}^{1}(\Omega)}<\infty$, and

(ii) $\sup _{t \geqslant \tau}\|u(\cdot, t)\|_{\infty}<\infty$ for any $\tau>0$.

Proof. Suppose that (i) is not true, then, due to Lemma 5.1,

$$
\lim _{t \rightarrow \infty} \inf \|\kappa(u(\cdot, t))\|_{H_{0}^{1}(\Omega)}<+\infty \quad \text { and } \quad \lim _{t \rightarrow \infty} \sup \|\kappa(u(\cdot, t))\|_{H_{0}^{1}(\Omega)}=+\infty
$$

must hold. According to Lemma 5.10 for an arbitrary large constant $B>\lim _{t \rightarrow \infty} \inf \Pi(t)$ there exists $w \in \omega(\psi)$ such that $\Pi[w]=\int_{\Omega} \mathrm{e}^{\kappa(w)} \mathrm{d} x=B^{2}$. Using the previous relation and the Gilbarg-Trudinger inequality we get

$$
B^{2} \leqslant C_{1}|\Omega| \mathrm{e}^{C_{2}\|\kappa(w)\|_{H_{0}^{1}(\Omega)}}
$$

and so $\|\kappa(w)\|_{H_{0}^{1}(\Omega)} \geqslant \ln \left(B^{2} / C_{1}|\Omega|\right)^{C_{1}}$. Also, Lemma 5.2 implies that there exists a constant $M=M(\psi)$ such that $\|\kappa(w)\|_{H_{0}^{1}(\Omega)} \leqslant M$. The above relations imply that $B \leqslant \sqrt{C_{1}|\Omega| \mathrm{e}^{\lambda / C_{2}}}$, which contradicts the fact that $B$ is an arbitrary large constant.

The main result of this section is as follows.

Proposition 5.13. Let $N=1, \Omega=(-1,1)$ or $N=2, \Omega=B(0,1)$ and $\psi(x) \in H_{0}^{1}(\Omega)$. If $\lambda>\lambda^{*}$ and $\kappa(s)$ is a function such that all the previous results are valid, then the solution $u(x, t ; \psi)$ of (5.1) blows up in finite time.

Proof. If $u(x, t ; \psi)$ is a global-in-time solution of problem (5.1), then, according to Propositions 5.9 and 5.12, $\sup _{t \geqslant 0}\|\kappa(u(\cdot, t))\|_{H_{0}^{1}(\Omega)}<\infty$ and $\sup _{t \geqslant \tau}\|u(\cdot, t)\|_{\infty}<\infty$ hold for $\tau>0$. The latter implies the existence of a steady-state solution $w \in \omega(\psi)$ of (5.1), which is a contradiction since for $\lambda>\lambda^{*}$ there are no steady states. Hence there exists a sequence $\left(t_{n}\right)_{n \in \mathbb{N}}$ with $t_{n} \rightarrow t_{\max }$ as $n \rightarrow \infty$ such that $\left\|\kappa\left(u\left(\cdot, t_{n}\right)\right)\right\|_{H_{0}^{1}(\Omega)} \rightarrow \infty$ and $\left\|u\left(\cdot, t_{n}\right)\right\|_{\infty} \rightarrow \infty$ as $n \rightarrow \infty$. According to Lemma 5.1 we have that $t_{\max }<\infty$, so the solution $u$ blows up in finite time. This completes the proof.

\section{Discussion}

In this work, the behaviour of solutions to the equation

$$
u_{t}=\Delta(\kappa(u))+\frac{\lambda f(u)}{\left(\int_{\Omega} f(u) \mathrm{d} x\right)^{p}}, \quad \Omega \subset \mathbb{R}^{N}, \quad N=1,2,
$$

associated with boundary and initial conditions, is studied mainly in the case where $f(s)=\mathrm{e}^{\kappa(s)}$. Some existence and non-existence results are given concerning the corresponding steady-state equation. Using energy methods, it is investigated under what 
circumstances the solution $u(x, t)$ is global in time or blows up in finite time. Moreover, when $0<p<1$ a blow-up result is proved, for dimensions $N=1,2$.

However, an open problem remains, which is the asymptotic behaviour of $u(x, t)$ in the case where $f(s) \neq \mathrm{e}^{\kappa(s)}$. In this case a Lyapunov functional cannot be constructed and so the method used to prove blow-up, when $0<p<1$ and $\lambda>\lambda^{*}$, fails.

\section{References}

1. R. A. Adams, Sobolev spaces (Academic, 1975).

2. D. G. ARONSON, On the Green's function for second order parabolic equations with discontinuous coefficients, Bull. Am. Math. Soc. 69 (1963), 841-847.

3. J. W. BEBERNES AND R. ELY, Existence and invariance for parabolic functional equations, Nonlin. Analysis 7 (1983), 1225-1235.

4. J. W. BeBernes AND A. A. LACEY, Global existence and finite-time blow-up for a class of nonlocal parabolic problems, Adv. Diff. Eqns 2 (1997), 927-953.

5. J. W. Bebernes and P. TAlaga, Nonlocal problems modelling shear banding, Commun. Appl. Nonlin. Analysis 3 (1996), 79-103.

6. M. FilA, Boundedness of global solutions of nonlinear diffusion equations, J. Diff. Eqns 98 (1992), 226-240.

7. M. Fila, Boundedness of global solutions of nonlocal parabolic equations, Nonlin. Analysis 30 (1997), 877-885.

8. D. Gilbarg And N. S. TRudinger, Elliptic partial differential equations of second order, 2nd edn (Springer, 1993).

9. N. I. Kavallaris and D. E. Tzanetis, Blow-up and stability of a nonlocal diffusionconvection problem arising in ohmic heating of foods, Diff. Integ. Eqns 15(3) (2002), $271-288$.

10. A. A. LACEY, Global blow-up of a nonlinear heat equation, Proc. R. Soc. Edinb. A 102 (1986), 305-313.

11. O. A. Ladyzenskaja, V. A. Solonnikov and N. N. Ural'Ceva, Linear and quasilinear equations of parabolic type, Translations of Mathematical Monographs, vol. 23 (Providence, RI, American Mathematical Society, 1968).

12. G. M. Lieberman, Study of global solutions of parabolic equations via a priori estimates, Part II, Porous medium equations, Commun. Appl. Nonlin. Analysis 1(3) (1994), 93-115.

13. D. E. Tzanetis And P. M. Vlamos, A nonlocal problem modelling ohmic heating with variable thermal conductivity, Nonlin. Analysis Real World Applic. 2(4) (2001), 443-454.

14. D. E. Tzanetis and P. M. Vlamos, Some interesting special cases of a nonlocal problem modelling ohmic heating with variable thermal conductivity, Proc. Edinb. Math. Soc. 44 (2001), 585-595. 